\title{
Effect of hydrogen on the slip resistance of tungsten single crystals
}

\author{
W.Z. Yao ${ }^{a}$, P. Wang ${ }^{a}$, A. Manhard ${ }^{a}$, C.E. Krill III ${ }^{b}$, J.H. You ${ }^{a *}$ \\ ${ }^{a}$ Max Planck Institute for Plasma Physics, EURATOM Association, Boltzmanstr.2, \\ 85748 Garching, Germany \\ bUlm University, Institute of Micro and Nanomaterials, Albert-Einstein-Allee 47, \\ 89081 Ulm, Germany
}

\begin{abstract}
The intense influx of hydrogen plasma onto the tungsten wall of a nuclear fusion reactor causes severe microstructural damage in the near-surface layer. The evolution of hydrogen-induced damage is often promoted by local plastic flow. Since hydrogen solutes are known to lower the hardness of many metals, the question arises as to the extent to which the tungsten walls are softened by the implantation of hydrogen. In this study, we investigated the change in slip resistance of tungsten single crystals following deuterium implantation. To determine the inherent yield stress of tungsten, we performed nanoindentation on high-purity single crystals. The statistical distribution of pop-in stresses (yield stress upon a single slip) revealed a significant reduction in pop-in load upon hydrogen implantation. In addition, the average stress at pop-in was found to be a function of crystallographic orientation of the samples, with much larger pop-in loads observed on (100) and (110) surface planes than on (111) surfaces.
\end{abstract}

*Corresponding author: Phone: +49 893299 1373; E-mail: you@ipp.mpg.de

Keywords: Tungsten; Hydrogen effect; Nanoindentation; Single crystals; Pop-in stress; Deuterium implantation 


\section{Introduction}

Currently, tungsten is the most-favored cladding material for plasma-facing components in nuclear fusion reactors. The tungsten-armored wall must withstand an intense particle influx consisting of energetic ions of hydrogen $(\mathrm{H})$ isotopes. Under severe bombardment by $\mathrm{H}$ ions, the wall surface undergoes microstructural damage formation, such as voids, blisters and cracks [1, 2], and the evolution of this damage is often accompanied by local plastic flow. In many metals, hydrogen solutes are known to reduce the hardness, leading to so-called hydrogen-enhanced localized plasticity [3, 4]. The $\mathrm{H}$ isotopes being implanted into and retained in the tungsten wall are thought to modify the plastic properties of tungsten. Consequently, the extent to which $\mathrm{H}$ solutes cause local softening of tungsten may be a key to understanding the peculiar damage patterns observed in hydrogen-irradiated tungsten armor.

Since such hydrogen-induced damage occurs at the length scale of individual grains, the effect of hydrogen on the plasticity of tungsten must be investigated at a comparable length scale. To the authors' knowledge, there are no reports of previous studies of this kind in the literature. The motivation of the present work is to demonstrate the change in inherent slip resistance of tungsten after an increase in $\mathrm{H}$ concentration. There are two technical challenges that must be overcome in order to accomplish this goal: first, figuring out how to identify the inherent slip resistance, and second, raising the local hydrogen content in a tungsten specimen.

The first problem is related to the issue of suppressing the secondary hardening effect caused by the interaction of dislocations with grain boundaries. To this end, we employed high-purity single crystals (SC) and the nanoindentation test, since the volume of the plastically deformed region during nanoindentation is comparable to the size of typical damage zones observed in tungsten walls.

The second problem arises from the fact that the equilibrium solubility of hydrogen in defect-free tungsten is extremely low, while the diffusivity is quite high $[5,6]$. The existence of crystal defects can increase the amount of retained $\mathrm{H}$ [6]. In general, single crystals grown from a melt have a very low dislocation density and, thus, few trapping sites for $\mathrm{H}$ solutes. This situation is obviously disadvantageous for the detection of hydrogen-induced effects in monocrystalline tungsten. Therefore, we used ion implantation from a deuterium (D) plasma to overcome the thermodynamic barrier to hydrogen entry in tungsten.

A characteristic feature commonly observed during nanoindentation tests of single crystals is the so called pop-in phenomenon, which refers to a sudden burst of penetration [7]. It is detected in the form of a staircase-like displacement excursion in 
the load-displacement curve during the early stage of a load-controlled nanoindentation test. Such pop-in events are peculiar to defect-free single crystals or large, recrystallized grains. Pop-in behavior has already been observed in tungsten $[8,9]$. The pop-in effect is believed to be caused by uniform nucleation and/or rapid multiplication (avalanche) of dislocations, leading to a sharp hardness drop and enhanced slip in the vicinity of the indented domain [10-13]. This interpretation is based on the observation that the resolved shear stress required to trigger a pop-in event is close to the theoretical strength of the material, $G / 2 \pi$, where $G$ is the shear modulus [14]. Furthermore, increasing the number of glissile dislocations leads to a decrease in pop-in load or even extinction of the pop-in effect [12]. The critical resolved shear stress needed for initiating a slip event can be estimated from the corresponding pop-in load. Hence, the effects of $\mathrm{H}$ solutes on the slip resistance can be inferred from the change in pop-in load as a function of hydrogen concentration.

In the present paper, we present statistical data for pop-in loads measured before and after implantation of $D$ into three different orientations of SC tungsten. Advanced analytical techniques are employed to quantify the low hydrogen concentration in the specimens with sufficient accuracy to reveal a clear trend in the pop-in load distribution with increasing hydrogen content.

\section{Experiment}

\subsection{Specimen preparation}

SC tungsten specimens were prepared in the form of rods having diameters of 6 or $9 \mathrm{~mm}$ from commercial material provided by Goodfellow Cambridge Limited. The manufacturer quoted a purity of better than $99.99 \mathrm{wt} \%$, which is roughly ten times better than the purity of other standard commercial products. It was reported that commercial SC tungsten with a purity of $99.9 \mathrm{wt} \%$ has a $\mathrm{H}$ impurity content ranging from 0.02 to 0.1 at\% $[15,16]$. Hence, it is reasonable to assume that the $\mathrm{H}$ content in the as-delivered SC tungsten samples used in this study was less than 0.01 at $\%$. The SC rod samples had a crystal growth axis in [100], [110] or [111] directions. For each crystal orientation, two disks ( $1 \mathrm{~mm}$ thick) were sliced along the rod axis using spark erosion. Thus, the surface normal of the sliced disks coincided with the [100], [110] or [111] crystal direction. To determine the orientation of the specimens, X-ray Laue backscattering measurements were performed before and after the cutting operation, ensuring that the orientation error was kept within $\pm 1.5^{\circ}$. 
Measuring pop-in loads is a tricky matter, as the nanoindentation test is highly sensitive to the quality of surface finishing [8]. Mechanical polishing is likely to cause plastic deformation of a surface layer due to abrasive wear. To avoid this artifact, the potentially damaged surface layer was removed by means of electrochemical polishing in an aqueous solution of $1.5 \% \mathrm{NaOH}$ at $25 \mathrm{~V}$ for $5 \mathrm{~min}$. Characterization by atomic force microscopy (AFM) showed that this etching treatment produced a smooth surface with typical roughness of 3 to $10 \mathrm{~nm}$. It is highly likely that a thin oxide film forms on the electrochemically etched surface of tungsten [17]; such surface oxide layers can affect the results of nanoindentation tests [18]. In addition, an oxide film can act as a barrier against $\mathrm{D}$ implantation, inhibiting the influx of $\mathrm{H}$ ions into a tungsten crystal. Tungsten trioxide $\left(\mathrm{WO}_{3}\right)$ begins to sublimate at $750^{\circ} \mathrm{C}$ and becomes strongly volatile above $900^{\circ} \mathrm{C}$ [19]. Thus the $\mathrm{WO}_{3}$ film can be removed by heating above $900^{\circ} \mathrm{C}$. In this work, the specimens were heated at $950^{\circ} \mathrm{C}$ for 12 hours under high-vacuum conditions $\left(10^{-5} \mathrm{mbar}\right)$. This high-temperature annealing step was also needed for removing preexisting $\mathrm{H}$ impurities. The specimen was transferred to the indentation device after furnace cooling in vacuum atmosphere.

\subsection{Hydrogen content before $D$ implantation}

For quantitative measurement of the $\mathrm{H}$ content in the bulk of the specimens, we employed thermal desorption spectroscopy (TDS). The TDS technique delivers information on the integral amount of $H$ or $D$ retained in a specimen, except for a small portion of the strongly bound solute atoms. During a TDS analysis, the trapped $\mathrm{H}$ and $\mathrm{D}$ solutes are released from the specimen in gaseous form during continuous heating and monitoring by a quadrupole mass spectrometer (QMS). Infrared radiation was used to heat the $\mathrm{SC}$ specimens from $20^{\circ} \mathrm{C}$ to $1150^{\circ} \mathrm{C}$ in a quartz tube at an average rate of $0.25 \mathrm{~K}$ per second. The 2 atomic mass unit signal (e.g. $\mathrm{H}_{2}$ molecules) dominated the release spectra.

Fig. 1 shows the signal spectra recorded for the released $\mathrm{H}_{2}$ molecules. The red spectrum indicates the amount of hydrogen contained in the as-polished specimen, whereas the blue spectrum denotes the hydrogen content of the specimen following vacuum-annealing at $950^{\circ} \mathrm{C}$. The fractional concentration of $\mathrm{H}$ per tungsten atom is estimated to be approximately $1.65 \times 10^{-5}$ in the as-polished specimen and $6.90 \times 10^{-6}$ in the annealed one. Evidently, the high-temperature annealing step lowered the $\mathrm{H}$ impurity content by roughly $60 \%$, reaching the ppm range. 


\subsection{Deuterium implantation}

For D implantation, we employed a calibrated plasma source (PlaQ) installed at the Max-Planck-Institute for Plasma Physics (IPP) in Garching. Technical details of the device are given elsewhere [20]. PlaQ was equipped with an electron-cyclotron resonance plasma generator. The mass distribution of the impinging $D$ ions was measured using an energy-selective mass spectrometer at the specimen position. For the given plasma conditions, the beam consisted mostly of $\mathrm{D}_{3}^{+}$ions $(94 \%)$, with a minor fraction of $\mathrm{D}_{2}^{+}$and $\mathrm{D}^{+}$(3\% each). To modify the kinetic energy of the ions, the specimen holder was biased with a DC voltage of $-100 \mathrm{~V}$.

The flux and energy of the impinging $D$ ions were measured using a differentially pumped retarding field analyzer. At the given discharge pressure of $1 \mathrm{~Pa}$ and nominal microwave power of $150 \mathrm{~W}$, the average energy of the $\mathrm{D}_{3}^{+}$ions was about $115 \mathrm{eV}$, which is equivalent to a mean energy of $38 \mathrm{eV}$ per $\mathrm{D}^{+}$atom. Such a low ion energy was desired to suppress the production of lattice defects by implantation. The D flux was $9 \times 10^{19} \mathrm{D} \mathrm{m}^{-2} \mathrm{~s}^{-1}$, and all specimens were exposed to the same total fluence of $6 \times 10^{24} \mathrm{D} \mathrm{m}^{-2}$. An actively cooled copper heat sink was used as specimen holder to hold the specimen temperature at $\sim 100^{\circ} \mathrm{C}$. After implantation, the specimens were immediately moved into a vacuum desiccator to prevent exposure to atmosphere.

\subsection{Deuterium content after $D$ implantation}

Once the implantation process ceases, the dynamic chemical equilibrium immediately switches over to static equilibrium. Several previous experimental determinations of the $D$ implantation profile in polycrystalline tungsten show that $D$ ions are enriched mostly in a near-surface layer of submicron depth [16, 21-23].

The $D$ concentration depth profile after implantation was measured by means of nuclear reaction analysis (NRA) using a comparable tungsten specimen. A fully recrystallized and annealed polycrystalline specimen with reduced defect density was employed (annealed at $1730^{\circ} \mathrm{C}$ for 30 minutes, average grain size: $216 \pm 10 \mu \mathrm{m}^{2}$, dislocation density: $1.9 \pm 1.4 \times 10^{12} \mathrm{~m}^{-2}$ ). The number density of available trap sites for $\mathrm{D}$ was comparable to the case of the SC samples. The recrystallized tungsten specimen was subjected to the same D implantation conditions as the SC specimens. Hence, the resulting $D$ concentration profile should be nearly the same as in the SC specimens. 
NRA was carried out at the Tandem ion accelerator at IPP. The $D\left({ }^{3} \mathrm{He}, \alpha\right) \mathrm{H}$ nuclear reaction-with ${ }^{3} \mathrm{He}$ projectile particle energy of $0.69 \mathrm{MeV}$-was used for near-surface analysis. The particles were energy-analyzed with a small-angle surface barrier detector at a scattering angle of $102^{\circ}$. The depth resolution of the detector was $21 \mathrm{~nm}$. The SIMNRA code was used to convert the original signal spectrum into a concentration depth profile [24].

The NRA data revealed that the implanted $D$ atoms were mostly concentrated within a very thin surface layer of about $20 \mathrm{~nm}$ depth. The maximum concentration reached 5 at\%, which is four orders of magnitude greater than the $\mathrm{H}$ impurity level of the annealed specimens. The relative error of this measurement was $2.7 \%$. The predicted penetration depth of $D$ implantation was calculated to be $8-10 \mathrm{~nm}$ by the Monte Carlo code TRIM [25, 26]. Beyond the surface layer, the D concentration decreases rapidly to below $0.01-0.001$ at $\%$, which is comparable to the background concentration level.

Recall that the solubility of deuterium in tungsten is extremely low while the diffusion rate of $D$ is quite high. Deuterium can exist stably only at trap sites (e.g. dislocations), either preexisting or created by implantation. Thus, the $\mathrm{D}$ concentration peak detected by NRA implies a certain population of defects in the near-surface layer. It should be noted that the kinetic energy of the injected $D$ ions $(38 \mathrm{eV})$ is far below the threshold energy required for producing any defect. On the other hand, the D plasma was found to be contaminated by a small amount of oxygen impurity. Heavy oxygen ions might have produced a very thin $(8 \mathrm{~nm})$ amorphous layer on the surface during $D$ implantation [26]. The nanoindentation tests are likely to be affected by the presence of such an amorphous surface layer. However, the influence of the latter on the pop-in phenomenon is thought to be insignificant, since the thickness of this layer is 25 times smaller than the typical range of pop-in displacement.

Furthermore, it should be noted that NRA can detect only $D$ atoms. If trap sites existed prior to implantation, $\mathrm{H}$ impurities may have already been captured at the preexisting trap sites prior to implantation. It is therefore probable that the total amount of $H$ and $D$ retained in the sample is greater than the $D$ content detected by NRA.

\subsection{Nanoindentation test}

Nanoindentation tests were conducted at room temperature using an instrumented nanoindentation device manufactured by Micro Materials Ltd (Wrexham, UK). Three-sided Berkovich indenters were used. The area function of the indenter as well as the machine compliance was carefully calibrated prior to our 
measurements. A total of 30 indentation tests were carried out in load-controlled mode for each specimen orientation and $D$ concentration, with the maximum load being increased from $10 \mathrm{mN}$ to $100 \mathrm{mN}$ and from $2 \mathrm{mN}$ to $10 \mathrm{mN}$. The loading rate was fixed at $0.5 \mathrm{mN} / \mathrm{s}$ and $0.1 \mathrm{mN} / \mathrm{s}$, correspondingly, and a dwell time of $10 \mathrm{~s}$ was inserted between the loading and unloading stages of each indentation. The sample hardness was estimated using the method of Oliver and Pharr [27].

\section{Results and discussion}

\subsection{Load-displacement curves and pop-in behavior}

Fig. 2(a) shows typical loading and unloading curves recorded during Berkovich nanoindentation performed on the (100) surface plane of an as-polished SC tungsten specimen. In this figure, ten arbitrarily selected curves are superimposed, the peak load of which increases from 10 to $100 \mathrm{mN}$ at an increment of $10 \mathrm{mN}$. The loading part of the curves manifests nearly perfect coincidence, indicating excellent reproducibility of the tests. The curves commonly exhibit a pronounced pop-in behavior during the early stage of indentation. Prior to the onset of a pop-in event, the deformation was purely elastic.

The low-load regime of the load-displacement curves of Fig. 2(a) is plotted at higher magnification in Fig. 2(b), which shows the pop-in behavior more clearly. The pop-in events are triggered at loads between 5 and $10 \mathrm{mN}$. The extent of abrupt penetration during pop-in ranged from $120 \mathrm{~nm}$ to $200 \mathrm{~nm}$, depending on the load at which pop-in was triggered, with the pop-in displacement increasing proportionally to the pop-in load. Fig. 2(b) manifests considerable scatter in the values of the pop-in loads. The first unloading curve obtained directly after a pop-in event reveals a significant amount of permanent penetration, which is comparable to the associated pop-in displacement. This finding is consistent with the conventional interpretation of pop-in phenomena as being caused by the homogeneous nucleation and collective glide of dislocations on discrete slip planes. The nanoindentation curves obtained on the remaining specimens showed qualitatively similar features, even for different crystal orientations.

Mechanically polished SC tungsten exhibits no pop-in effect at all, owing to the ample number of dislocations produced by plastic wear [8]. The pop-in behavior evident in Fig. 2 therefore indicates that the damaged surface layer of our specimens was effectively removed by electrochemical polishing. 
Fig. 3 shows the early stage of loading and unloading curves obtained by nanoindentation performed on the (100) surface plane of an annealed SC tungsten specimen. The nanoindentation response of the annealed specimen looks almost the same as that of the as-polished one: the range of the pop-in loads and pop-in displacements was barely affected by the high-temperature heat treatment. This implies that the initial hydrogen impurity level was sufficiently low that subsequent reduction of the hydrogen concentration by annealing caused no change in the slip resistance.

The initial elastic regime of the indentation curves in Fig. 2 and 3 can be described by the Hertz theory of contact mechanics [28, 29]. The elastic response of an isotropic material subjected to spherical indentation (i.e. Hertzian contact) is given by

$$
P=\frac{4}{3} E_{r} R^{1 / 2} h^{3 / 2},
$$

with $P$ denoting the indentation load, $h$ the penetration displacement, $R$ the effective indenter tip radius, and $E_{r}$ the reduced elastic modulus, which is defined by

$$
\frac{1}{E_{r}}=\frac{1-\mathrm{v}_{i}^{2}}{E_{i}}+\frac{1-\mathrm{v}^{2}}{E},
$$

where $E_{i}$ and $E$ represent the elastic modulus of the diamond indenter and of tungsten, respectively, and $v_{i}$ and $v$ are the Poisson's ratio of diamond and tungsten, respectively. The estimated value for $E_{r}$ is $321 \mathrm{GPa}$, which was obtained from Eq. (2) and the parameter values $E_{i}=1141 \mathrm{GPa}, E=410 \mathrm{GPa}, v_{i}=0.07$ and $v=0.28$ [27]. The average value of $R$ was determined to be $675 \mathrm{~nm}$ by means of numerical curve fitting of Eq. (1) to the initial elastic loading part of the loaddisplacement curve, as proposed by Bei et al. [14].

Fig. 4 shows the pop-in regime of the loading and unloading curves obtained on a D-implanted SC tungsten specimen with (100) surface plane. In contrast to the previous cases (Fig. 2 and 3), the D-implanted specimens exhibit less-pronounced pop-in behavior. Both the pop-in loads and the pop-in displacements were reduced remarkably by deuterium implantation; in fact, several of the indentation curves show no pop-in behavior at all.

In the present case, it should be pointed out that the location of maximum shear stress, where dislocations are supposed to be nucleated most probably, is quite distant from the near-surface domain where implanted $D$ atoms are populated. The position of maximum shear stress (i.e. the most probable position of slip initiation) at the moment of pop-in event is estimated to be $122-153 \mathrm{~nm}$ in the hydrogen-free 
specimens and 10-133 $\mathrm{nm}$ in the D-implanted specimens. The implanted D atoms were concentrated within the depth of $20 \mathrm{~nm}$. A question arises as to how the implanted $D$ atoms confined in the surface layer could promote slip taking place at significantly deeper positions.

One possible explanation may be the forced diffusion of $D$ atoms being expelled from the original positions of decoration at defects due to the mechanical perturbation under indentation. The $D$ atoms are progressively released from the defects being stressed by the penetrating indenter tip. The released $D$ atoms diffuse outward and combine again with neighboring dislocations. Simultaneously, the D atoms reduce the activation energy of slip leading to premature pop-in.

In Fig. 5, the distributions of measured pop-in loads are shown for the (100) surface plane of as-polished, annealed and D-implanted specimens. Each histogram is constructed from the data of thirty independent tests. All three histograms manifest a considerable degree of scatter in the pop-in loads. Such a stochastic nature of popin stresses was previously reported by Morris et al. [30].

Although the distributions of pop-in loads for the as-polished and annealed samples look quite similar, the same cannot be said for the pop-in loads recorded on the D-implanted specimen. An essential difference is the overall decrease in pop-in load following $D$ implantation. The maximum pop-in load of the D-implanted sample was $7 \mathrm{mN}$, but most pop-in loads were less than $5 \mathrm{mN}$. In contrast, the pop-in loads of the as-polished and annealed specimens lay in the range of 4 to $10 \mathrm{mN}$. The distributions plotted in Fig. 5 (a) and (b) nearly overlap, indicating a negligible effect of annealing on pop-in behavior. Recalling that one of the aims for vacuum annealing was to remove any surface oxide film that might have formed, we conclude from the lack of influence of annealing on pop-in behavior that the latter has nothing to do with the fracture of an oxide film; rather, the pop-in stresses and displacements must be a consequence of dislocation slip, as discussed by Gerberich [11].

One of the physically meaningful measures for evaluating the slip resistance of a single crystal is the critical resolved shear stress projected onto the primary slip system [7]. The critical resolved shear stress denotes the shear stress acting in a slip plane when plastic yield (slip) takes place. Gerberich et al. [7] calculated the coefficients of the tensor transformation equation that relates the stress fields generated by a Hertzian contact to the resolved shear stresses on the (112)[11 $\overline{1}]$ and (121) [11 $\overline{1}]$ slip systems. They showed that, for a given load, the largest resolved shear stress occurs directly underneath the center of a spherical indenter tip of radius $a$ at the depth $0.48 a$. At this location, the resolved shear stress is $0.31 p_{m}$, 
where $p_{m}$ denotes the maximum pressure generated under the indenter [7] for the indentation load $P$. This pressure is given by [29]

$$
p_{m}=\left(\frac{6 P E_{r}^{2}}{\pi^{3} R^{2}}\right)^{\frac{1}{3}} .
$$

The shear stress $\tau_{p}$ at which pop-in occurs can be calculated by

$$
\tau_{p}=0.31 p_{p}=0.31\left(\frac{6 P_{p} E_{r}^{2}}{\pi^{3} R^{2}}\right)^{\frac{1}{3}}
$$

where $p_{p}$ and $P_{p}$ denote the maximum pressure and the indentation load at a popin event, respectively.

The measured pop-in loads were converted into shear stresses using Eq. (4). The estimated shear stress values for the pop-in loads are displayed along the top axis in Figs. 5, 6, and 7. For the as-polished and annealed specimens, the shear stresses for pop-in range from $17 \mathrm{GPa}$ to $23 \mathrm{GPa}$. This result agrees roughly with the theoretical prediction reported by Roundy et al. [31]: according to their density functional analysis, the ideal yield stress of monocrystalline tungsten under shear was calculated to be $18 \mathrm{GPa}$, taking on identical values for slip occurring on $\{110\}$, $\{112\}$ and $\{123\}$ planes. In the case of the D-implanted sample, the values for shear stress at pop-in range from 0 to $20 \mathrm{GPa}$, but they are mostly smaller than 18 GPa.

In Fig. 6, the distributions of measured pop-in loads are plotted for the (110) surface of as-polished, annealed and D-implanted SC tungsten specimens. The shear stress values are indicated along the upper abscissa. Clear differences are evident between Figs. 5 and 6: for example, the histograms for the (110) surface of unimplanted samples manifest greater scattering than do the samples with a (100) surface. In addition, the distribution of pop-in shear stresses is broader and more uniform for the (110) surface, extending into the domain of lower stresses. In the case of the D-implanted samples, the range of pop-in shear stresses for the (110) surface was shifted to lower values, mostly below $15 \mathrm{GPa}$.

Fig. 7 shows the distributions of pop-in loads for SC tungsten specimens with a (111) surface. In this case, we find that the pop-in shear stresses are much smaller than for the two other surface orientations. In addition, the distributions of pop-in stresses are much narrower than in the previous cases. Owing to the overall decrease in pop-in stress, the impact of $D$ implantation appears to be weak, but it is still discernable, as all values for the pop-in stress of the D-implanted sample lie 
below $13 \mathrm{GPa}$, whereas this quantity takes on slightly larger values in the case of the unimplanted samples.

Comparison of Figs. 5, 6 and 7 reveals a significant dependence of the pop-in load distributions on surface orientation. For all cases of specimen treatment (aspolished, annealed and D-implanted), the data exhibited the highest pop-in load range for the (100) orientation, a somewhat decreased range for (110) surfaces, and considerably lower pop-in loads for the (111) orientation. An orientation dependence for pop-in loads was also reported for single crystals of gold [32].

In all cases of sample orientation tested so far, the overall effect of $D$ implantation seems to be clear and consistent: D implantation significantly reduces the pop-in loads.

In literature, similar effects were also reported by Barnoush and Vehoff $[33,35]$ and Barnoush et al.[34], where hydrogen was electrochemically infiltrated into the specimens. By means of in-situ nanoindentation tests in electrolyte solutions, they demonstrated that hydrogen reduced the pop-in stresses of copper, aluminum, iron and nickel. This feature was attributed to the effect of hydrogen-enhanced local plasticity. Hydrogen atoms facilitate homogeneous dislocation nucleation by reducing dislocation line energy and thus the activation energy for dislocation nucleation.

\subsection{Hardness}

The hardness data determined by nanoindentation are summarized in Table 1. The error range of the hardness values is relatively small, indicating a low degree of scatter in the present tests. Such a high reproducibility of the plastic response during nanoindentation is a characteristic feature of tungsten single crystals. According to Miyahara and Yamamoto [36], this feature can be attributed to uniformity in plastic properties on a nanometer length scale.

The values collected in Table 1 offer no evidence for a significant dependence of hardness on surface orientation-in contrast to the previous finding that the distribution of pop-in loads depends considerably on orientation. Likewise, hardness was scarcely affected by $\mathrm{D}$ implantation as expected, since the D-implanted layer was simply too thin to generate any effect on the plastic deformation extending over several hundred nanometers. This is evident in the nanoindentation curves of Fig. 8, which compare load-displacement curves recorded on the (100) surface of SC tungsten both prior to $D$ implantation and afterwards. Beyond the first yielding event (that is, after the first pop-in), both curves exhibit full overlap during the loading as well as unloading stages. 


\section{Summary and Conclusions}

This manuscript presents statistical data for pop-in stresses during nanoindentation of tungsten single crystals before and after D implantation. Highpurity specimens were electrochemically polished and vacuum-heated $\left(950^{\circ} \mathrm{C}, 12 \mathrm{~h}\right)$ prior to $D$ implantation to reduce the hydrogen impurity concentration and to remove any oxide films. Thermal desorption spectroscopy showed that the annealing step reduced the $\mathrm{H}$ concentration in the bulk from $1.65 \times 10^{-3}$ at $\%$ to $6.90 \times 10^{-4}$ at $\%$. Deuterium was implanted into tungsten with low ion energy (38 eV) up to a fluence of $6 \times 10^{24} \mathrm{D} \mathrm{m}^{-2}$. The depth profile of $\mathrm{D}$ concentration measured by nuclear reaction analysis revealed that the implanted $D$ solutes were located in a surface layer of about $20 \mathrm{~nm}$ thickness at a local concentration up to 5 at\%.

A clear correlation between the range of pop-in loads and the $D$ content was found, with the average pop-in load decreasing significantly after $D$ implantation for all crystal orientations. On the other hand, the distributions of pop-in loads of the aspolished and annealed specimens exhibited no significant differences. These findings provide clear evidence for hydrogen-induced plastic softening.

In addition, the distribution of critical pop-in stresses was found to be a function of crystal orientation. For all cases of specimen treatment (as-polished, annealed and D-implanted), pop-in loads exhibited a consistent trend in orientation dependence: the (100) orientation showed the highest pop-in load range and the (111) orientation the lowest. In contrast to the pop-in loads, the hardness values showed neither orientation dependence nor a change following $D$ implantation as expected.

\section{Acknowledgements}

The authors gratefully acknowledge their colleague, Th. Dürbeck, for his friendly support in performing the TDS measurement. One of the authors (W.Z. Yao) is also grateful to the Chinese Scholarship Council (CSC) for financial support.

\section{Reference}

[1] M. Balden, S. Lindig, A. Manhard, J.H. You, J. Nucl. Mater., 414 (2011) 69-72.

[2] S. Lindig, M. Balden, V.K. Alimov, A. Manhard, C. Hoschen, T. Hoschen, B. Tyburska-Puschel, J. Roth, Phys. Scr., T145 (2011) 7. 
[3] H.K. Birnbaum, P. Sofronis, Mater. Sci. Eng. A, 176 (1994) 191-202.

[4] H. Vehoff, Hydrogen Related Material problems, Hydrogen in Metals III, Springer, Berlin, 1997, pp. 215-278.

[5] R. Frauenfelder, J.Vac.Sci.Technol. 6 (1969) 388-397.

[6] D.F. Johnson, E.A. Carter, J. Mater. Res., 25 (2010) 315-327.

[7] W.W. Gerberich, J.C. Nelson, E.T. Lilleodden, P. Anderson, J.T. Wyrobek, Acta Mater., 44 (1996) 3585-3598.

[8] D.F. Bahr, D.E. Kramer, W.W. Gerberich, Acta Mater., 46 (1998) 3605-3617.

[9] S.A.S. Asif, J.B. Pethica, Philos. Mag. A, 76 (1997) 1105-1118.

[10] D. Lorenz, A. Zeckzer, U. Hilpert, P. Grau, H. Johansen, H.S. Leipner, Phys. Rev. B, 67 (2003) 4.

[11] W.W. Gerberich, D.E. Kramer, N.I. Tymiak, A.A. Volinsky, D.F. Bahr, M.D. Kriese, Acta Mater., 47 (1999) 4115-4123.

[12] M.A. Lodes, A. Hartmaier, M. Göken, K. Durst, Acta. Mater., 59 (2011) 42644273.

[13] C. Begau, A. Hartmaier, E.P. George, G.M. Pharr, Acta Mater., 59 (2011) 934942.

[14] H. Bei, E.P. George, J.L. Hay, G.M. Pharr, Phys. Rev. Lett., 95 (2005) 045501.

[15] A.A. Haasz, M. Poon, R.G. Macaulay-Newcombe, J.W. Davis, J. Nucl. Mater., 290-293 (2001) 85-88.

[16] V.K. Alimov, J. Roth, R.A. Causey, D.A. Komarov, C. Linsmeier, A. Wiltner, F. Kost, S. Lindig, J. Nucl. Mater., 375 (2008) 192-201.

[17] J. Garnaes, F. Kragh, K.A. Morch, A.R. Tholen, J. Vac. Sci. Technol., A 8(1990) 441-444.

[18] D.E. Kramer, K.B. Yoder, W.W. Gerberich, Philos. Mag. A, 81 (2001) 2033-2058.

[19] E. Lassner, W. D. Schubert, Tungsten: Properties, Chemistry, Technology of the Element, Alloys, and Chemical Compounds, Springer, 1999.

[20] A. Manhard, T. Schwarz-Selinger, W. Jacob, Plasma Sources Sci. Technol., 20 (2011) 9.

[21] M. Fukumoto, H. Kashiwagi, Y. Ohtsuka, Y. Ueda, M. Taniguchi, T. Inoue, K. Sakamoto, J. Yagyu, T. Arai, I. Takagi, T. Kawamura, J. Nucl. Mater., 390-391 (2009) 572-575.

[22] V.K. Alimov, J. Roth, M. Mayer, J. Nucl. Mater., 337-339 (2005) 619-623.

[23] B. Tyburska, V.K. Alimov, O.V. Ogorodnikova, K. Schmid, K. Ertl, J. Nucl. Mater., 395 (2009) 150-155.

[24] M. Mayer, IPP Report 9/113, Max-Planck-Institut für Plasmaphysik, Garching, Germany, 1997. 
[25] W. Eckstein, IPP Report 9/132, Max-Planck-Institut für Plasmaphysik, Garching, Germany, 2002.

[26] A. Manhard, University of Augsburg, Augsburg, 2012.

[27] W.C. Oliver, G.M. Pharr, J. Mater. Res., 7 (1992) 1564-1583.

[28] H. Hertz, J. Reine. Angew. Math. 92 (1882) 156-171.

[29] K.L. Johnson, Contact Mechanics, Cambridge Press, Cambridge, 1985.

[30] J.R. Morris, H. Bei, G.M. Pharr, E.P. George, Phys. Rev. Lett., 106 (2011) 4.

[31] D. Roundy, C.R. Krenn, M.L. Cohen, J.W. Morris, Philos. Mag. A, 81 (2001) 1725-1747.

[32] J.D. Kiely, J.E. Houston, Phys. Rev. B, 57 (1998) 12588.

[33] A. Barnoush, H. Vehoff, Acta Mater., 58 (2010) 5274-5285.

[34] A. Barnoush, M. Asgari, R. Johnsen, Scr. Mater., 66 (2012) 414-417.

[35] A. Barnoush, H. Vehoff, Scr. Mater., 55 (2006) 195-198.

[36] K. Miyahara, T. Yamamoto, Recent Advancement of Theory and Practice in Hardness Measurement, Tsukuba, Japan, 2007, pp. 21-24. 


\section{Figure captions}

Fig. 1. Thermal desorption spectra of hydrogen release from single crystal tungsten specimens before and after annealing (at $950^{\circ} \mathrm{C}$ for 12 hours in a highvacuum furnace). The hydrogen release rates were normalized for comparison between as-polished and annealed specimens.

Fig. 2. Typical load-displacement curves of Berkovich nanoindentation tests carried out on the (100) plane of an electrochemically polished single crystal tungsten. (a) Loading and unloading curves for incrementally increased indentation loads; (b) low-load regime of the indentation curves showing pop-in displacements (jumps in displacement at constant applied load).

Fig. 3. Low-load regime of nanoindentation curves measured on the (100) plane of an annealed SC tungsten specimen.

Fig. 4. Low-load regime of nanoindentation curves measured on the (100) plane of a D-implanted SC tungsten specimen.

Fig. 5. Distribution of measured pop-in loads on (100) planes of (a) as-polished, (b) annealed, and (c) D-implanted SC tungsten specimens. Each histogram contains data from thirty nanoindentation tests.

Fig. 6. Distribution of measured pop-in loads on (110) planes of (a) as-polished, (b) annealed, and (c) D-implanted SC tungsten specimens. Each histogram contains data from thirty nanoindentation tests.

Fig. 7. Distribution of measured pop-in loads on (111) planes of (a) as-polished, (b) annealed, and (c) D-implanted SC tungsten specimens. Each histogram contains data from thirty nanoindentation tests.

Fig. 8. Load-displacement curves of nanoindentation tests carried out on the (100) plane of SC tungsten before (solid symbols) and after (open symbols) D implantation. 


\section{Figures}

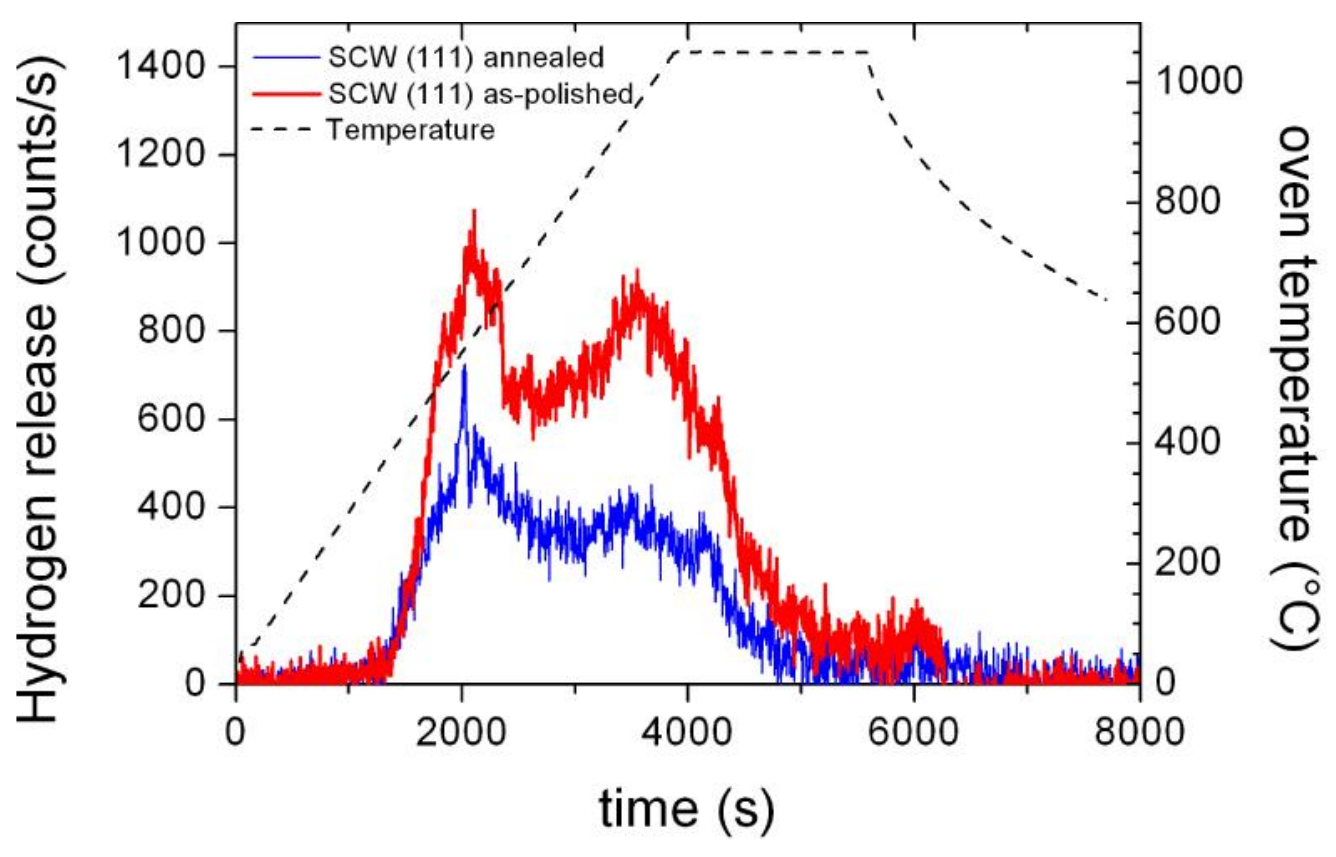

Fig. 1. Thermal desorption spectra of hydrogen release from single crystal tungsten specimens before and after annealing (at $950^{\circ} \mathrm{C}$ for 12 hours in a high-vacuum furnace). The hydrogen release rates were normalized for comparison between as-polished and annealed specimens. 


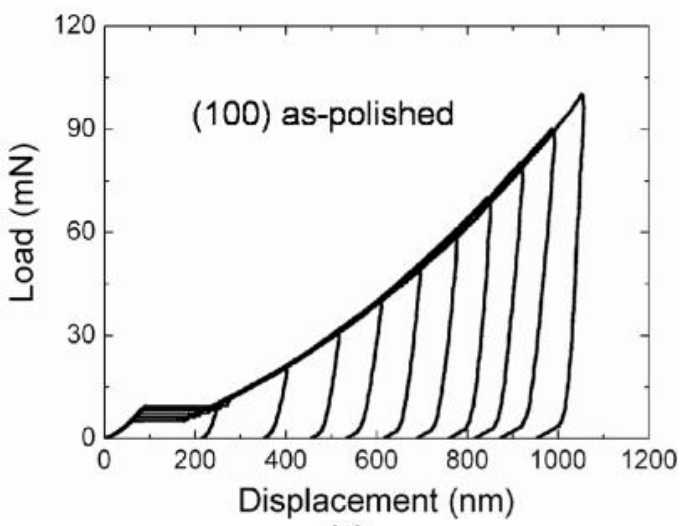

(a)

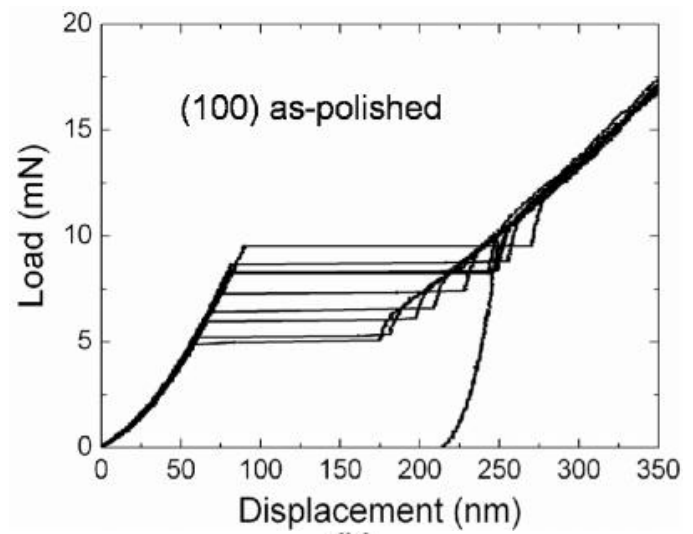

(b)

Fig. 2. Typical load-displacement curves of Berkovich nanoindentation tests carried out on the (100) plane of an electrochemically polished single crystal tungsten. (a) Loading and unloading curves for incrementally increased indentation loads; (b) low-load regime of the indentation curves, showing pop-in displacements (jumps in displacement at constant applied load). 


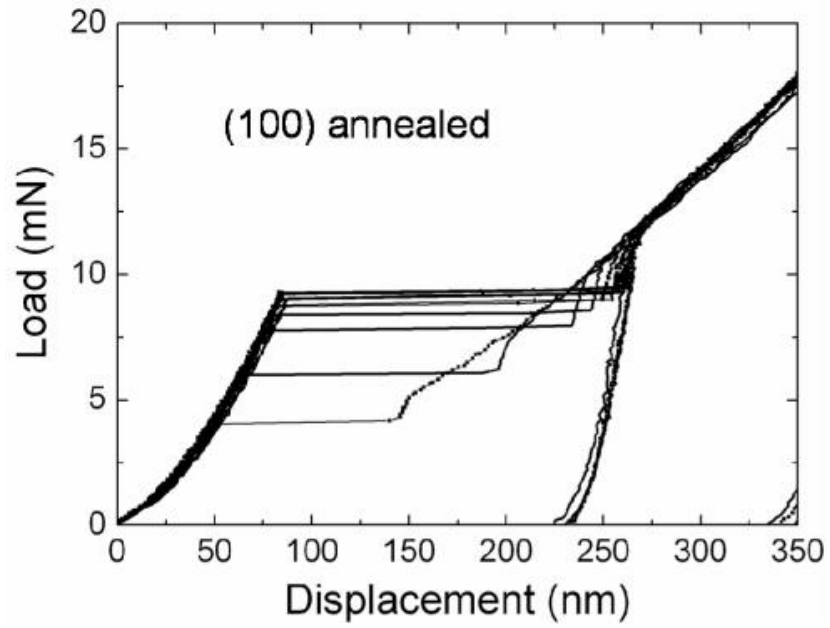

Fig. 3. Low-load regime of nanoindentation curves measured on the (100) plane of an annealed SC tungsten specimen. 


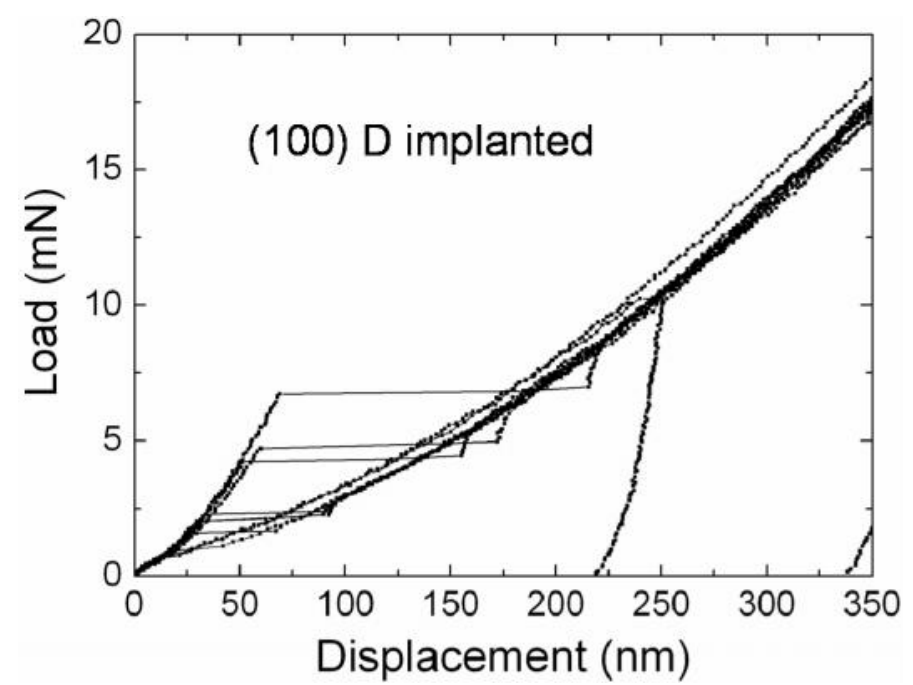

Fig. 4. Low-load regime of nanoindentation curves measured on the (100) plane of a Dimplanted SC tungsten specimen. 


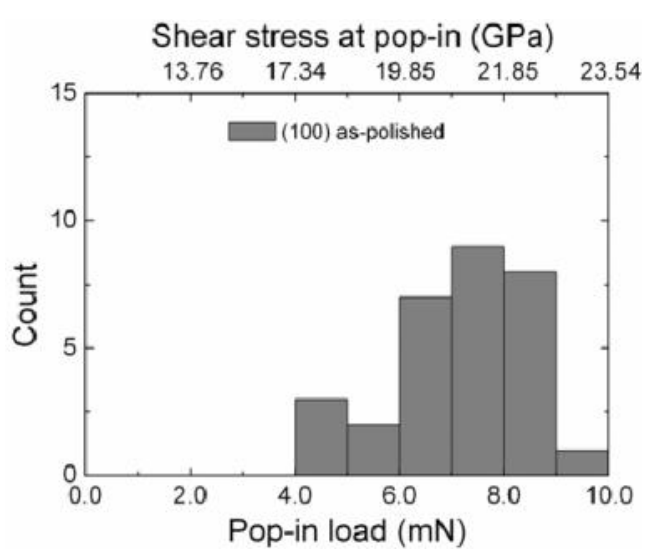

(a)

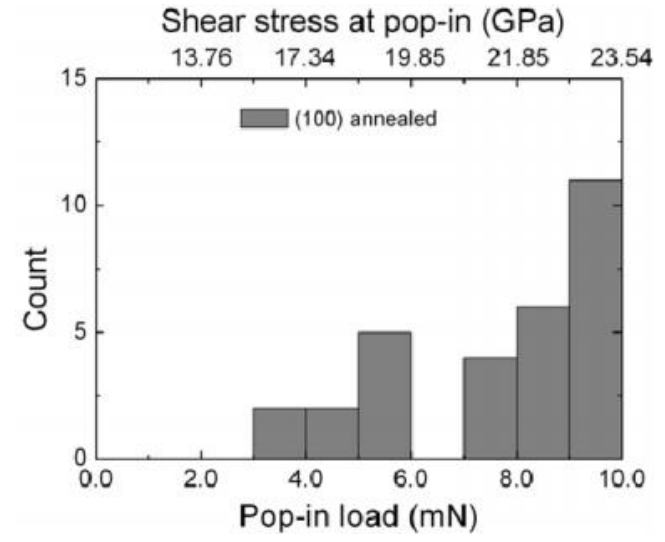

(b)

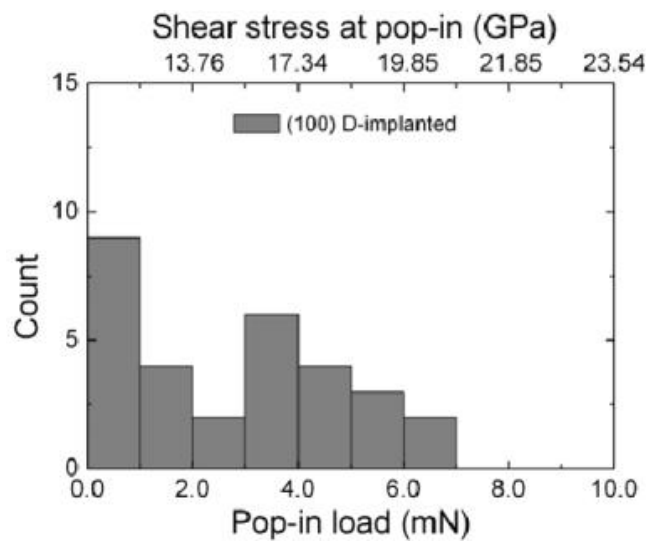

(c)

Fig. 5. Distribution of measured pop-in loads on (100) planes of (a) as-polished, (b) annealed, and (c) D-implanted SC tungsten specimens. Each histogram contains data from thirty nanoindentation tests. 


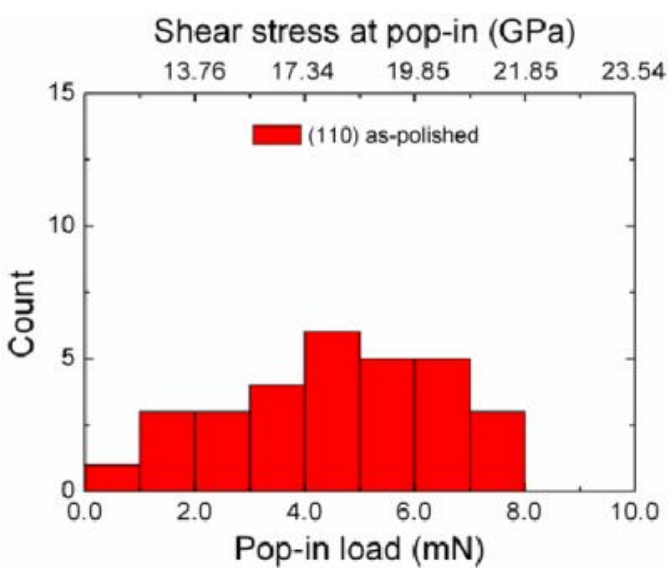

(a)

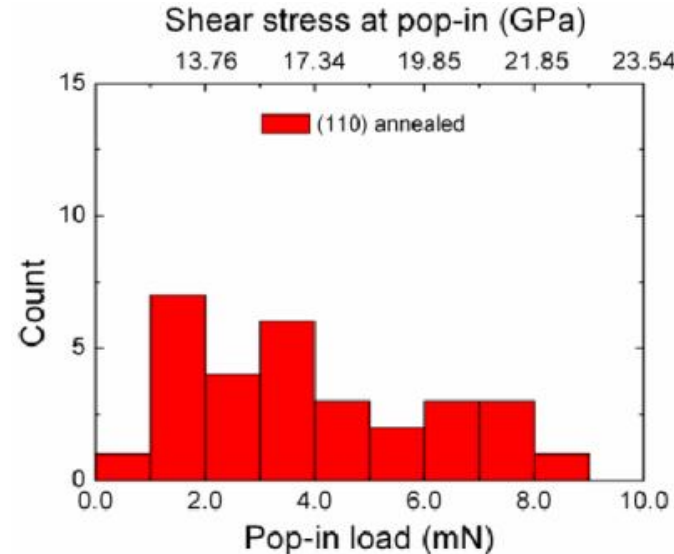

(b)

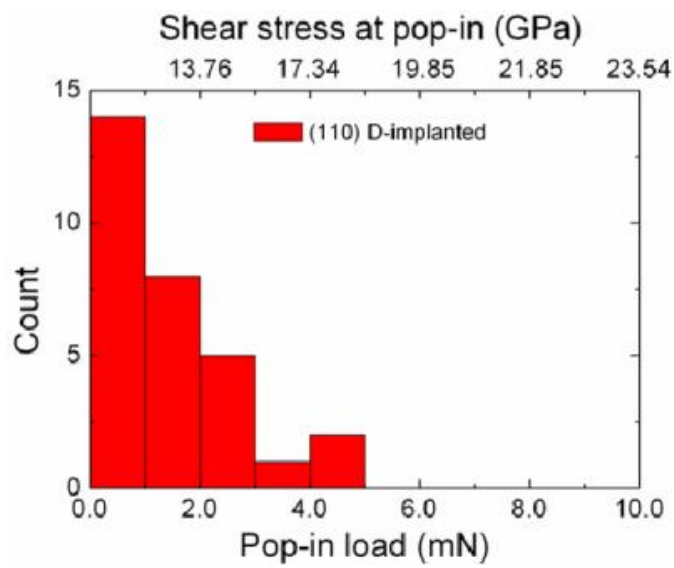

(c)

Fig. 6. Distribution of measured pop-in loads on (110) planes of (a) as-polished, (b) annealed, and (c) D-implanted SC tungsten specimens. Each histogram contains data from thirty nanoindentation tests. 


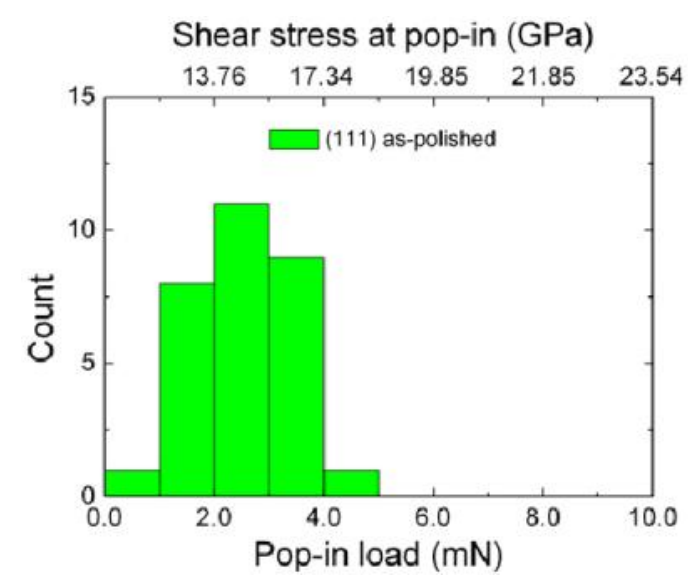

(a)

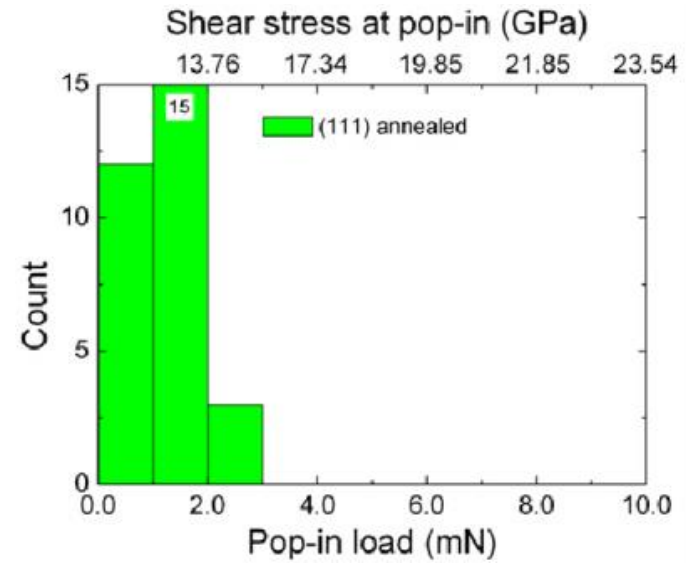

(b)

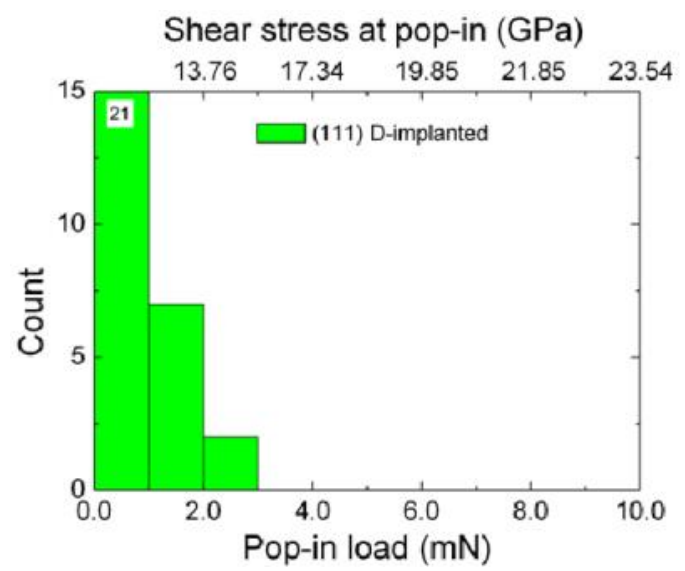

(c)

Fig. 7. Distribution of measured pop-in loads on (111) planes of (a) as-polished, (b) annealed, and (c) D-implanted SC tungsten specimens. Each histogram contains data from thirty nanoindentation tests. 


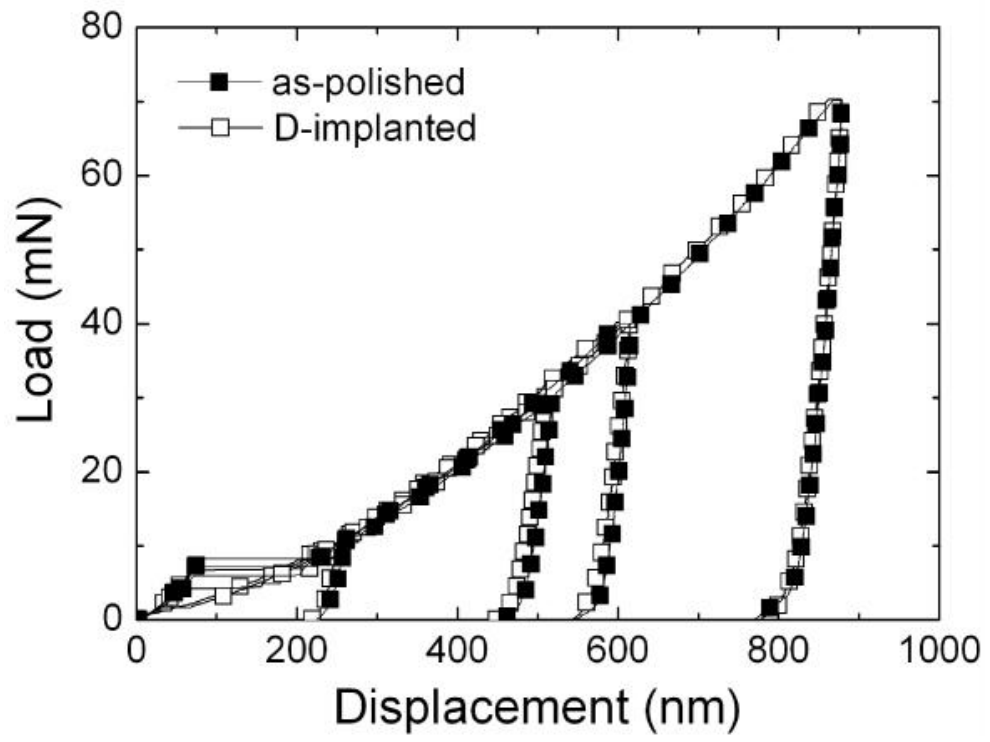

Fig. 8. Load-displacement curves of nanoindentation tests carried out on the (100) plane of SC tungsten before (solid symbols) and after (open symbols) D implantation. 
Table 1. Hardness values for tungsten single crystals, as determined from nanoindentation measurements.

\begin{tabular}{cccc} 
Surface orientation & as-polished (GPa) & annealed (GPa) & D-implanted (GPa) \\
\hline$(100)$ & $4.46 \pm 0.22$ & $4.49 \pm 0.14$ & $4.44 \pm 0.15$ \\
$(110)$ & $4.41 \pm 0.15$ & $4.43 \pm 0.12$ & $4.38 \pm 0.12$ \\
$(111)$ & $4.44 \pm 0.09$ & $4.47 \pm 0.11$ & $4.42 \pm 0.21$
\end{tabular}

